УДК: 618.177:618.145-007.415-08 МЕСТО ГОРМОНАЛЬНОЙ ТЕРАПИИ В ПРЕОДОЛЕНИИ БЕСПЛОДИЯ,
АССОЦИИРОВАННОГО С ТЯЖЕЛЫМИ ФОРМАМИ ЭНДОМЕТРИОЗА

\author{
(C) Д.А. Геркулов
}

ГБОУ ВПО «Первый Санкт-Петербургский государственный медицинский университет им. акад. И.П. Павлова» Минздрава РФ

- Актуальность. Преодоление бесплодия при эндометриозе представляет собой одну из наиболее трудных проблем репродуктивной медицины. Целью явилось изучение эффективности протоколов ЭКО у больных, оперированных по поводу эндометриом яичников, при использовании различных вариантов адъювантной терапии. Материалы и меmоды. В исследование включены 144 пациентки, страдающие бесплодием, после оперативного лечения эндометриом яичников, разделенные на 3 группы в зависимости от варианта адъювантной гормональной терапии перед протоколом ЭКО: антагонистами ГнРГ, диеногестом 2 мг в сутки и без лечения. Результаты. Частота клинической беременности в протоколах ЭКО у больных после терапии диеногестом 2 мг в сутки в 2,5 раза (44,7 и 16,7\% соответственно; $p=0,01)$, а частота родов — в 3 раза выше $(36,8$ и 11,1 \% соответственно, $p=0,01)$ по сравнению с таковой у пациенток без гормонального лечения. Выводы. Результат проведенного исследования позволяет считать диеногест в дозировке 2 мг в сутки эффективным средством гормональной терапии тяжелых форм генитального эндометриоза перед протоколом ЭКО.

- Ключевые слова: эндометриоз; эндометриома; ЭКО; ICSI; диеногест.

\title{
ROLE OF ADJUVANT HORMONAL THERAPY IN TREATMENT OF ENDOMETRIOSIS-ASSOSIATED INFERTILITY
}

\author{
(c) D. A. Gerkulov \\ I.P. Pavlov First State Medical University of St. Petersburg, Russia
}

- Background. In vitro fertilization (IVF) represents the most efficient means of overcoming endometriosis-related infertility. Objective. To investigate the outcomes of in vitro fertilization cycles after controlled ovarian hyperstimulation in patience operated for endometrioma after different adjuvant hormonal therapy. Material and methods. 144 patients with endometriosisassociated infertility undergoing IVF were divided into three groups: GnRH agonist administration; dienogest 2 mg daily administration; women without adjuvant therapy. Results. Clinical pregnancy rates were 2,5 times higher $(44,7$ and $16,7 \%$ respectively; $p=0,01)$ and live birth rate was 3 times higher $(36,8$ и 11,1\% respectively; $p=0,01)$ in dienogest administration group before IVF/ICSI cycle in comparison with no adjuvant therapy groups, respectively. Conclusion. Considering the clinical pregnancy and live birth rate rates dienogest administration may be effective in patients with mild-to-moderate endometriosis prior to IVF/ICSI cycle.

- Key words: endometriosis; endometrioma; IVF; ICSI; dienogest.

Актуальность преодоления бесплодия у больных наружным гениальным эндометриозом обусловлена высокой частотой встречаемости заболевания, мультифакториальностью природы нарушения репродуктивной функции, высокой частотой необоснованных повторных оперативных вмешательств на органах малого таза, планированием беременности в позднем репродуктивном возрасте, а также поздним использованием вспомогательных репродуктивных технологий [5].

Наиболее трудной задачей представляется преодоление бесплодия у пациенток с тяжелыми формами эндометриоза, в частности с эндометриомами яичников [6].

Предполагается, что в основе механизмов снижения эффективности протоколов ЭКО лежат две основные причины: снижение качества и количества получаемых ооцитов, а также нарушение рецептивности эндометрия $[8,10]$. Согласно данным литературы применение адъювантной гормональной терапии агонистами ГнРГ (аГнРГ) в течение 3-6 месяцев перед ЭКО увеличивает эффективность протоколов в 4 раза (ОШ: 4,28; $95 \%$ ДИ, 2,0-9,15) [13].

Однако необходимость супрессивной гормональной терапии не ограничивается лишь повышением частоты наступления беременности. Лечение эндометриоза перед планируемой беременностью необходимо для снижения рисков развития осложнений, повышенных при эндометриозе: невынашивания беременности, гестоза, преждевременных родов, гипотрофии плода [3].

Целью настоящего исследования явилось изучение эффективности протоколов ЭКО и исходов беременностей у больных после оперативного лечения эндометриом яичников. 


\section{Материалы и методы}

В исследование были включены 144 пациентки с эндометриоз-ассоциированным бесплодием после оперативного лечения эндометриом яичников. Средний возраст пациенток составил $33 \pm 4$ года (от 23 до 42 лет). Индекс массы тела не превышал $30 \mathrm{\kappa г} / \mathrm{M}^{2}$. У всех обследованных больных на момент вступления в протокол ЭКО уровень ФСГ был менее $12,0 \mathrm{ME} /$ мл $(7,56 \pm 2,55 \mathrm{ME} /$ мл), уровень АМГ был не ниже 0,5 нг/мл.

В зависимости от варианта адъювантной гормональной терапии перед вступлением в протокол ЭКО (ЭКО/ICSI) все исследуемые пациентки были разделены на 3 группы. В I группу вошли 38 пациенток, получавших диеногест 2 мг в сутки в течение 3-6 месяцев, II группу составили 70 пациенток, получавших препараты аГнРГ в течение 3-6 месяцев, III группу составили 36 женщин, не получавших гормональную терапию НГЭ перед протоколом ЭКО. Группы были сопоставимы по возрасту, состоянию овариального резерва, длительности бесплодия, индексу массы тела.

С целью стимуляции суперовуляции использовался гибкий протокол с использованием антагонистов ГнРГ. Стартовая доза рекомбинантных гонадотропинов подбиралась индивидуально в зависимости от возраста и состояния овариального резерва пациентки. Антагонист ГнРГ назначали при достижении лидирующим фолликулом диаметра 14 мм. Критерием введения триггера финального созревания фолликулов было достижение 3 фолликулами (при развитии 3 и более фолликулов) или 1 фолликулом (при развитии менее 3 фолликулов) диаметра $17 \mathrm{mм.}$ Оплодотворение ооцитов осуществлялось с помощью ЭКО или ЭКО +ICSI. Перенос эмбрионов производился на 3-й, 4-й или 5-й день культивирования. Максимальное количество переносимых эмбрионов не превышало 2. Эффективность протокола оценивали с помощью показателя частоты имплантации (с помощью оценки концентрации $\beta$-субъединицы хорионического гонадотропина в крови на 14-й день после переноса эмбрионов) и частоты клинической беременно- сти (визуализация плодного яйца в полости матки). Статистическая обработка данных выполнена с помощью электронных таблиц Microsoft Excel и пакета прикладных программ Statistica for Windows. 7, StatSoftInc. (США). Все значения указаны как среднее (M) \pm стандартное отклонение (SD).

\section{Результаты исследования}

Исходы протоколов ЭКО оценивались по частоте наступления беременности (ЧНБ) на протокол ЭКО и частоте наступления беременности на перенос эмбрионов, частоте родов на протокол ЭКО. Всего перенос эмбрионов был осуществлен в $79,9 \%$ случаев. Общая ЧНБ на протокол ЭКО составила $32,6 \%$.

При расчете частоты наступления беременности в зависимости от адъювантной терапии были получены значимые различия (табл. 1). В первой группе беременность наступила в 44,7\% случаев, во второй группе в 34,3 и в $16,7 \%$ в третьей группе исследования $\left(\chi^{2}=6,793, \mathrm{p}=0,033\right)$. Статистически значимые отличия выявлены между I (диеногест) и III (без гормональной терапии) группами, $p=0,012$. Шансы наступления беременности в группе с использованием диеногеста в качестве адъювантной терапии в 2,5 раза выше, чем без гормональной терапии $(\mathrm{OШ}=4,04$; $95 \%$ \%И 1,37-11,94, p=0,015). Несмотря на отсутствие статистической значимости, у пациентов, получавших аГнРГ в качестве гормональной терапии перед протоколом ЭКО, частота наступления беременности в 2 раза превышала таковую у пациентов без гормональной терапии (ОШ $=2,61$; $95 \%$ ДИ: $1,56-4,37, p=0,072)$.

При расчете на перенос эмбрионов частота наступления клинической беременности в I группе составила $48,6 \%$, во II $-42,1 \%$, в III $-26,1 \%$ $\left(\chi^{2}=2,975, \mathrm{p}=0,226\right)$. Несмотря на отсутствие статистической достоверности, ЧНБ в ІІІ группе была в полтора раза ниже, чем во II группе (ОШ 2,79, $95 \%$ ДИ 1,64-4,74) и почти в два раза ниже, чем в первой группе (ОШ 3,62, 95 \% ДИ 1,19-11,06). Анализ частоты имплантации среди исследуемых групп не показал статистически значимых разли-

Таблица 1

Частота наступления беременности у больных исследуемых групп (в расчете на протокол ЭКО)

\begin{tabular}{|c|c|c|c|c|c|c|c|c|c|c|}
\hline \multirow[b]{2}{*}{ Характеристика } & \multicolumn{3}{|c|}{ I группа $(\mathrm{n}=38)$} & \multicolumn{3}{|c|}{ II группа (n=70) } & \multicolumn{3}{|c|}{ III группа (n=36) } & \multirow{2}{*}{$\begin{array}{c}\text { P-level, } \\
\mathrm{df}=2\end{array}$} \\
\hline & Абс. & $\begin{array}{c}\text { Отн., } \\
\%\end{array}$ & $\begin{array}{c}95 \%, \\
\text { ДИ (\%) }\end{array}$ & Абс. & $\begin{array}{c}\text { Отн., } \\
\%\end{array}$ & $\begin{array}{c}95 \%, \\
\text { ДИ(\%) }\end{array}$ & Абс. & $\begin{array}{c}\text { Отн., } \\
\%\end{array}$ & $\begin{array}{c}95 \%, \\
\text { ДИ(\%) }\end{array}$ & \\
\hline $\begin{array}{c}\text { Частота } \\
\text { наступления } \\
\text { клинической } \\
\text { беременности }\end{array}$ & $17 / 38$ & 44,7 & $26,4-63,1$ & $24 / 70$ & 34,3 & $21,9-46,8$ & $6 / 36$ & 16,7 & $1,8-31,6$ & $\begin{array}{c}\mathrm{p}_{\mathrm{I}-\mathrm{II}}=0,309 \\
\mathrm{p}_{\mathrm{I}-\mathrm{III}}=0,015^{*} \\
\mathrm{p}_{\mathrm{II}-\mathrm{III}}=0,072\end{array}$ \\
\hline
\end{tabular}


Таблица 2

Частота прерывания беременности до 12 недель

\begin{tabular}{|c|c|c|c|c|c|c|c|}
\hline \multirow{2}{*}{ Характеристика } & \multicolumn{2}{|c|}{ I группа (n=17) } & \multicolumn{2}{|c|}{ II группа (n=24) } & \multicolumn{2}{|c|}{ III группа (n=6) } & \multirow{2}{*}{$\begin{array}{c}\text { P-level, } \\
\mathrm{df}=2\end{array}$} \\
\hline & Абс. & Отн., \% & Абс. & Отн., \% & Абс. & Отн., \% & \\
\hline $\begin{array}{c}\text { Самопроизвольный } \\
\text { аборт до } 12 \text { нед. }\end{array}$ & 3 & 17,7 & 24 & 16,7 & 2 & 33,3 & $\mathrm{p}=0,371$ \\
\hline
\end{tabular}

чий. Однако в группе пациентов, принимавших диеногест 2 мг в сутки, частота имплантации в расчете на количество перенесенных эмбрионов составила $32,2 \%$ и была выше таковой во второй группе - $28,7 \%$ и значительно выше, чем в третьей группе пациентов - 17,1\%.

Анализ исходов беременностей показал более высокую частоту самопроизвольного прерывания беременности в группе без адъювантной гормональной терапии $(33,3 \%)$, что было значительно выше, чем в группе, получавшей диеногест $(17,7 \%)$ и группе, получавшей аГнРГ (16,7\%), хоть и не достигло статистической значимости, $\mathrm{p}=0,371$ (табл. 2).

При расчете частоты родов на протокол ЭКО в исследуемых группах выявлены статистически достоверные отличия (таблица 3). Частота родов в I группе $(36,8 \%, 95 \%$ ДИ $20,1-61,8)$ превышала частоту родов в III группе исследования $(11,1 \%, 3,0-28,5)$ в 3 раза (ОШ: $4,67,95 \%$ ДИ $1,36-15,98), \mathrm{p}_{1-3}=0,01$. Различия в частоте родов между II и III группами были значительны $(28,6$ и $11,1 \%$; ОШ 3,2, $95 \%$ ДИ 1,0-10,2), но не достигли достоверности $\left(\mathrm{p}_{2-3}=0,043\right.$ статистически не значимо с учетом поправки Бонферрони для трех групп). Различия в частоте родов между II и I группами были незначительны $\left(36,8\right.$ и $28,6 \%$ соответственно, $\left.\mathrm{p}_{1-2}=0,479\right)$.

Средняя масса новорожденных у женщин с эндометриомами яичников в анамнезе, беременность у которых наступила в результате ЭКО, составила 3190 г $(3050 ; 3520)$ при одноплодной беременности и 2825 г $(2685 ; 3050)$ при двойнях. Срок родоразрешения при одноплодной беременности $39(38 ; 39)$ недель, при двойнях - $37(37 ; 38)$ недель. Одна беременность завершилась преждевременными родами при сроке гестации
34 недели. Средний вес новорожденных при одноплодной беременности среди групп исследования достоверно не отличался и составил 3245,8 , 3292,0 и 3130 г для групп, получавших диеногест 2 мг в сутки, аГнРГ и без лечения соответственно, $p=0,432$. При двойнях значения также не различались достоверно $(2597,5,2939,5$ и 2855,0 г для I, II и III групп соответственно, $\mathrm{p}=0,567$ )

$74 \%$ беременных были родоразрешены путем операции кесарева сечения. Одна беременность родоразрешена путем операции кесарева сечения в экстренном порядке при сроке 34 недели в связи с преждевременным излитием околоплодных вод при тазовом предлежании плода. $7 \%$ операций кесарева сечения выполнены по экстренным показаниям в связи с развившейся острой гипоксией плода в I периоде родов. $89 \%$ беременных родоразрешены оперативным путем в плановом порядке по совокупности относительных показаний. Различий в частоте операций кесарева сечения среди групп не выявлено.

\section{Обсуждение}

В проведенном нами исследовании показано, что использование супрессивной гормональной терапии в течение 3-6 месяцев перед протоколом ЭКО позволяет повысить эффективность протокола. Причем частота клинической беременности у больных, получавших диеногест 2 мг в сутки, достоверно превышала аналогичный показатель у больных без гормональной терапии и была выше таковой у пациенток, использующих аГнРГ. Полученные данные могут быть объяснены особенностями центрального и периферического действия диеногеста. Слабое антигонадотропное действие диеногеста в дозировке 2 мг в сутки [4], с одной стороны, не подавляет фолликулогенез,

Частота родов в расчете на протокол ЭКО в исследуемых группах

\begin{tabular}{|c|c|c|c|c|c|c|c|c|c|c|}
\hline \multirow[b]{2}{*}{ Исход беременности } & \multicolumn{3}{|c|}{ I группа (n=38) } & \multicolumn{3}{|c|}{ II группа (n=70) } & \multicolumn{3}{|c|}{ III группа (n=36) } & \multirow{2}{*}{$\begin{array}{c}\text { p-level, } \\
\mathrm{df}=2\end{array}$} \\
\hline & Абс. & Отн., \% & $\begin{array}{c}95 \%, \\
\text { ДИ (\%) }\end{array}$ & Абс. & Отн., \% & $\begin{array}{c}95 \%, \\
\text { ДИ (\%) }\end{array}$ & Абс. & Отн., \% & $\begin{array}{c}95 \%, \\
\text { ДИ (\%) }\end{array}$ & \\
\hline $\begin{array}{c}\text { Частота } \\
\text { родов }\end{array}$ & 14 & 36,8 & $20,1-61,8$ & 20 & 28,6 & $17,5-44,1$ & 4 & 11,1 & $3,0-28,5$ & $\begin{array}{l}\mathrm{p}_{\mathrm{I}-\mathrm{II}}=0,479 \\
\mathrm{p}_{\mathrm{I}-\mathrm{III}}=0,01^{*} \\
\mathrm{p}_{\mathrm{II}-\mathrm{III}}=0,043\end{array}$ \\
\hline
\end{tabular}


в то же время противовоспалительный эффект (снижение активности макрофагов, экспрессии IL-1) [9] препятствует «выгоранию» яичника согласно теории Kitajima [10]. Возможно, эффект диеногеста обусловлен не только его влиянием на эктопическую ткань, но и не исключено положительное влияние препарата на эутопический эндометрий. Так Hayashi А и соавт. (2012) было показано, что использование диеногеста приводит к увеличению соотношения PR-B/PR-A и снижению соотношения $\mathrm{ER} \beta / \mathrm{ER} \alpha$, что обеспечивает снижение так называемой «прогестероновой резистентности» эндометрия, характерной для эндометриоза [7].

В нашем исследовании отмечено повышение частоты самопроизвольных прерываний беременности у пациентов, не получавших гормональную терапию эндометриоза. Это может быть связано с изменениями в клетках эндометрия на ранних этапах беременности и нарушениями в локальном иммунном ответе в эндометрии $[1,12]$.

В зарубежной литературе также отмечено увеличение рисков преждевременных родов (ОШ 2,24; 95\% ДИ, 1,46-3,44) [2], развития гестоза (ОШ 7,5; 95 \% ДИ, 1,7-33,3) [3], гипотрофии плода (ОШ 2,72; 95 \% ДИ, 1,46-5,06) [2], родоразрешения путем операции кесарева сечения (ОШ 1,93; 95 \% ДИ, 1,31-2,84) у пациенток с эндометриозассоциированным бесплодием в анамнезе [3]. Различий в массе родившихся детей среди групп в нашем исследовании не получено. Это может быть связано с незначительным количеством выборки и требует дальнейших исследований.

Таким образом, гормональная терапия эндометриоза у женщин, страдающих бесплодием, перед вступлением в протокол ЭКО может быть рекомендована как с целью увеличения шансов наступления клинической беременности, так и для снижения рисков невынашивания и поздних осложнений беременности.

Статья представлена М.И. Ярмолинской, ФГБНУ «НИИ АГИР им. Д.О. Отта», Санкт-Петербург

\section{Литература}

1. Benagiano G., Brosens I., Habiba M. Structural and molecular features of the endomyometrium in endometriosis and adenomyosis. Hum. Reprod. Update. 2014; 20: 386-402.

2. Brosens I.A., de Sutter P., Hamerlynck T., Imeraj L., Yao Z., Cloke B. et al. Endometriosis is associated with a decreased risk of pre-eclampsia. Hum. Reprod. 2007; 22: 1725-9.

3. Conti N., Cevenini G., Vannuccini S., Orlandini C., Valensise H., Gervasi M. T. et al. Women with endometriosis at first pregnancy have an increased risk of adverse obstetric outcome. J. Matern. Fetal Neonatal. Med. 2015; 28:1795-8.

4. Foster R. H., Wilde M. I. Dienogest. Drugs. 1998; 56 (5): 825-33.

5. Giudice L. C., Kao L.C. Endometriosis. Lancet. 2004; 364: 1789-99.

6. Gupta S., Agarwal A., Agarwal R., Loret de Mola J. R. Impact of ovarian endometrioma on assisted reproduction outcomes. Reprod. Biomed. Online. 2006; 13: 349-60.

7. Hayashi A., Tanabe A., Kawabe S., Hayashi M., Yuguchi H., Yamashita $Y$. et al. Dienogest increases the progesterone receptor isoform B/A ratio in patients with ovarian endometriosis. J. Ovarian. Res. 2012; 5: 31.

8. Kao L.C., Germeyer A., Tulac S., Lobo S., Yang J.P., TayIor R. N. et al. Expression profiling of endometrium from women with endometriosis reveals candidate genes for diseasebased implantation failure and infertility. Endocrinology. 2003; 144: 2870-81.

9. Katsuki Y., Takano Y., Futamura Y. et al. Effects of dienogest, a synthetic steroid, on experimental endometriosis in rats. Eur. J. Endocrinol. 1998; 138: 216-6.

10. Kitajima M., Dolmans M.M., Donnez O. et al. Enhanced follicular recruitment and atresia in cortex derived from ovaries with endometriomas. Fertil. Steril. 2014; 101 (4): 1031-7.

11. Lin H., Leng J.H., Liu J.T., Lang J.H. Obstetric outcomes in Chinese women with endometriosis: a retrospective cohort study. Chin. Med. J. 2015; 128: 455-8.

12. Petraglia F., Arcuri F., de Ziegler D., Chapron C. Inflammation: a link between endometriosis and preterm birth. Fertil. Steril. 2012; 98: 36-40.

13. Sallam H. N., Garcia-Velasco J.A., Dias S., Arici A. Long-term pituitary down-regulation before in vitro fertilization (IVF) for women with endometriosis. Cochrane Database Syst. Rev. 2006; 1: CD004635.

\section{- Адреса автора для переписки}

Геркулов Дмитрий Александрович — аспирант. ГБОУ ВПО «Первый Санкт-Петербургский государственный медицинский университет им. акад. И. П. Павлова» Минздрава РФ. 197022, Россия, СанктПетербург, ул. Л. Толстого, д. 6-8. E-mail: gerkulov@gmail.com.
Gerkulov Dmitriy Alexandrovich - post-graduate student. I.P. Pavlov First State Medical University of St. Petersburg, Department of obstetrics, gynecology and reproductology. 197022, St. Petersburg, L. Tolstoy St., 6-8, Russia. E-mail: gerkulov@gmail.com. 